\title{
Anti eotaxin-2 antibodies attenuate the initiation and progression of experimental atherosclerosis*
}

\author{
Adi Mor ${ }^{1 \#}$, Arnon Afek ${ }^{1}$ Michal Entin-Meer ${ }^{2}$, Gad Keren $^{2}$, Jacob George ${ }^{1}$ \\ ${ }^{1}$ ChemomAb Ltd., Tel Aviv, Israel \\ ${ }^{2}$ The Department of Cardiology, Tel Aviv Sourasky Medical Center, Tel Aviv, Israel \\ Email: \#adimor@chemomab.com
}

Received 21 May 2013; revised 30 June 2013; accepted 10 July 2013

Copyright (C) 2013 Adi Mor et al. This is an open access article distributed under the Creative Commons Attribution License, which permits unrestricted use, distribution, and reproduction in any medium, provided the original work is properly cited.

\begin{abstract}
Background: The chemokine eotaxin-2 is a potent chemoattractant for inflammatory cells, the predominants of which are eosinophils. Human and murine atherosclerotic plaques are known to exhibit inflammatory phenotypes where a complex interaction of cytokine and chemokines plays a role. We tested the hypothesis that eotaxin-2 (eo-2) plays a causative role in the initiation and progression of experimental atherosclerosis. Methods and Results: Sera collected from atherosclerotic ApoE knockout (KO) mice, exhibited significantly higher levels of eo-2 compared to sera collected from their background age matched C57BL/6 litters by ELISA. Moreover, levels of eo-2 were higher in old atherosclerotic ApoE KO mice than in young animals. Similarly, the expression level of the eo- 2 receptor, CCR3, was increased in splenocytes of old ApoE compared to the young littermates. Administration of polyclonal blocking antibodies to eotaxin-2 resulted in a significant reduction of early atherosclerotic plaques in ApoE KO mice whereas prolonged treatment of mice with advanced plaques led to atheroma stabilization. A monoclonal antibody (D8) prepared against eo-2 attenuated adhesion of lymphocytes to fibronectin and potently inhibited their migration towards VEGF. Monoclonal blocking antibodies to eo-2 also significantly reduced atherosclerotic plaques in ApoE KO mice. Conclusion: Eo-2 serum levels are elevated in sera of ApoE KO mice with experimental atherosclerosis and its blockade is associated with reduced fatty streak accumulation and increased plaque stabilization.
\end{abstract}

Keywords: Atherosclerosis; Vulnerable Plaque;

*Conflict of Interest Disclosures: None for all authors of the manuscript.

${ }^{\#}$ Corresponding author.
Inflammation; Eotaxin-2; Chemokines

\section{INTRODUCTION}

The CC chemokine eotaxin-2 (eo-2) binds the eosinophil receptor CCR3 and possesses a potent chemotactic activity for eosinophils, basophils and Th2-type lymphocytes [1-4]. The eotaxin-2/CCL24 receptor CCR3, a seventransmembrane receptor coupled to heterotrimeric G proteins, is expressed in endothelial cells [5-9] as well as in macrophages and brain microglia [10].

Atherosclerosis is a complex disorder involving interaction of cellular and humoral components [11]. Whereas initial stages of atherosclerosis are mediated by interactions between monocytes and endothelial cells, subsequent plaque growth is influenced by smooth muscle cells, fibroblasts and lymphocytes. Inflammation with involvement of different leukocyte subpopulations and secretion of cytokines and chemokines is thought to play a pivotal role in promoting atherosclerotic plaque growth and propensity to destabilize and subsequently rupture [12-15].

It has recently been demonstrated that allergic cellular components may be considered important in the athrosclerotic plaque [12]. In addition, circumstantial evidence suggests that eotaxin-2/CCL 24 may play an active role in the process. Thus, other than being expressed in several types of endothelial cells [5-7], CCR3 has been reported to be expressed in plaque macrophages and to some extent, in smooth muscle cells $[10,12]$. Further suggestive of an effect of eotaxin-2/CCL 24 on atherogenesis is the recent observation that it induces activation of the plaque destabilizing proMMP-2 and of EGF-receptor in smooth muscle cells [13]. These findings are reinforced by a clinical study showing that in a cohort of healthy men, a non-conservative polymorphism in the eotaxin- 2 gene is associated with increased risk for myocardial infarction [14]. Moreover, in a subsequent study, it has been found 
that increased circulating eo-2 levels are associated with the presence of coronary atherosclerosis and ischemia [15-18].

In a recent study, we have demonstrated a significant therapeutic potential of our newly-developed monoclonal antibody, D8, on the development of adjuvant-induced arthritis in rats [19]. The results prompted us to assume that D8 may represent a novel therapeutic target in rheumatoid arthritis as well as in additional inflammatory disorders including atherosclerosis.

By blocking the eo-2 pathway either by polyclonal or a newly-developed specific monoclonal antibody, we were able to demonstrate inhibition of fatty streak formation and plaque stabilization employing the ApoE knockout (KO) mouse model. The data described herein point to the putative key role which eo-2 may play in the initiation and progression of atherosclerosis.

\section{MATERIALS AND METHODS}

\subsection{Cell Culture}

HEK 293 parental cells were cultured in DMEM (Biological Industries, Israel) supplemented with $10 \%$ fetal calf serum (FCS) (Invitrogen) and 1\% penicillin/streptomycin sulfate (Biological Industries, Israel). The cells were stably transfected with pCDNA-CCR3 (the plasmid was a generous gift from Prof. S. Peiper, Medical College of Georgia, Georgia, USA), followed by two rounds of cell sorting using an anti-CCR3-PE-conjugated antibody (Bioledgend, San Diego, CA) to yield an 85\% CCR3 stably-transfected population. The cells were maintained at $37^{\circ} \mathrm{C}$ in a humid incubator with $5 \% \mathrm{CO}_{2}$.

\subsection{Mice}

ApoE-KO mice on a C57BL/6 background were purchased from Jackson laboratories, (Bar Harbor, ME). C57BL/6J mice were obtained from Harlan Laboratories, Jerusalem, Israel. The mice were maintained at the local animal facility.

\subsection{Eo-2 and Anti-Eo-2 Ployclonal Antibody}

Purified human eo-2 protein and a neutralizing polyclonal anti-eo-2 antibody were purchased from Peprotech, USA.

\subsection{Production of Monoclonal Antibodies Directed against Eotaxin-2}

We have produced several clones of monoclonal antibodies (mAbs) to eo-2, according to standard protocols. In short, Balb/C mice were immunized with 20 ug of eo-2 (Peprotec, USA) followed by four additional boosts. After confirming the presence of polyclonal anti-eo-2 Abs in the sera, the mice were sacrificed and their spleens hybridized with an NS/0 myeloma line, followed by clonal screening for binding to eotaxin-2. The hybriddomas were then grown in serum-free media for 2 - 3 weeks, media collected and loaded onto $100 \mathrm{kDa}$ centricons (Biological Industries, Israel) for antibody concentration.

\subsection{Adhesion Assays}

In adhesion assays, mice or rat splenocytes, as well as human PBMC were separated on ficoll gradient and plated in $10 \mathrm{~cm}$ dishes for an overnight incubation. On the next day, the cells were harvested and pretreated with $50 \mathrm{ug} / \mathrm{ml}$ of D8 (the chosen anti-eo-2 monoclonal antibody) or total mouse IgG for two hours with rotation. The cells were then centrifuged and plated on 96-well plates pre-coated with fibronectin. After one hour-incubation, non-adherent cells were washed away and the amount of adherent cells was analyzed using XTT kit (Biological Industries, Israel).

\subsection{Migration Assays}

C57BL/6-derived splenocytes, rat splenocytes and human PBMCs pretreated with increasing doses of D8 $(12.5,25$ and $50 \mathrm{ug} / \mathrm{ml})$ at a volume of $150 \mathrm{ul}$ were plated on the upper of an 8 um insert placed on the top of a well in a 24-well plate (Fisher Scientific, USA). The lower part of each well contained $600 \mathrm{ul}$ of serum-free media supplemented with VEGF $(20 \mathrm{ng} / \mathrm{ml})$. Four hours later the media in the lower chamber was collected and cells counted using flow cytometry.

\subsection{Inhibition of Eotaxin-2/CCR3 Interaction in the Presence of D8}

Human-CCR3 tansfected or untransfected HEK cells were plated in six replicates on 96-well plates $(100,000$ cells/well), pre-coated with a complex of eo-2 $(5 \mathrm{ug} / \mathrm{ml}) /$ D8 $(30 \mathrm{ug} / \mathrm{ml})$. After one hour-incubation, non-adherent cells were washed away and the amount of adherent cells was analyzed using XTT kit.

\subsection{The Effect of Eo-2 Blockade on Atherosclerosis in ApoE KO Mice}

For the experiments involving the commercial anti-eo-2 antibody, 6 weeks old (young) and 4 months old ApoE $\mathrm{KO}$ mice were i.p. injected twice weekly with 5 ug of commercial goat anti mouse eo-2, demonstrating an antichemoattractant activity according to the manufacture's data sheet (Perotech, USA), mouse IgG or PBS. The animals from each group were sacrificed 8 weeks after treatment start for assessment of atherosclerosis. For the study assessing the in vivo effects of the newly-produced monoclonal antibody, 6 weeks old ApoE KO mice were 
i.p. injected three times a week with 30 ug of the D8, total mouse $\operatorname{IgG}$ or with vehicle only (6 animals/arm). Eight weeks later, mice were sacrificed for assessment of atherosclerosis. The study was approved by the local institutional animal care committee.

\subsection{Lipid Profile}

Total plasma cholesterol and triglyceride levels were determined using an automated enzymatic technique (Boehringer Mannheim, Germany).

\subsection{Assessment of Atherosclerosis}

Quantification of atherosclerotic fatty streak lesions was done by calculation of lesions size in the aortic sinus as previously described

http://atvb.ahajournals.org/cgi/content/full/19/3/505-R22 \#R22 [17] with a few modifications. In brief, the heart and upper portion of the aorta were removed from the animals and the peripheral fat was carefully removed. The upper section was embedded in OCT medium and frozen. Every other section $(10-\mu \mathrm{m}$ thick) throughout the aortic sinus $(400 \mu \mathrm{m})$ was taken for analysis. The distal portion of the aortic sinus was recognized by the 3 valve cusps that form the junction of the aorta to the heart. Sections were evaluated for fatty streak lesions after being stained with oil red $\mathrm{O}$. Lesion areas per section were counted on a grid by an observer who was unfamiliar with the tested specimen. Staining with Masson's trichrome and Von giesson's were employed to determine fibrous area and the contents of elastic fibers, respectively. The proportional area of the plaque stained positive for collagenous fibrosis was determined by quantitative morphometry. Immunohistochemistry for detection of plaque $\mathrm{T}$ cells was performed using an anti-CD3 antibody (Serotek, USA) and that of plaque macrophage was done with anti-moma (Serotek, USA), or with an anti-eo-2 antibody (Peprotech, Israel) followed by an HRP-conjugated goat anti-mouse secondary antibody (Jackson, USA).

\subsection{Statistical Analysis}

All parameters were evaluated by the one way ANOVA or two-tailed student's t-test as indicated. $\mathrm{P}<0.05$ was considered statistically significant. Results are expressed as mean \pm SEM unless otherwise specified in the text. Data was processed by the GraphPad software.

\section{RESULTS}

\subsection{Differential Expression of Eotaxin-2 and CCR3 in Murine Atheromas and Sera}

Since eo-2 and its receptor CCR3 are abundantly expressed in immune cells, we sought to assess the possible involvement of this chemokine in atherosclerosis. To this end, we initially determined the levels of eo-2 in the sera of old ApoE KO mice (4 mice) which spontaneously develop atherosclerotic plaques, compared to their C57BL/ 6 wild-type littermates (4 animals). Levels of eo-2 detected by ELISA were twice higher in ApoE KO compared to $\mathrm{C} 57 \mathrm{BL} / 6$ mice ( $\mathrm{P}<0.05$; one way ANOVA). Furthermore, older atherosclerotic ApoE mice exhibited higher levels of the chemokine compared to their younger littermates ( 4 mice in each group) ( $\mathrm{P}<0.05$; ANOVA) (Figure 1(a)). Serum levels eo-2 were considerably higher in ApoE KO mice compared to C57BL/6 litters $(\mathrm{P}<$ 0.05 ; ANOVA). Moreover a flow cytometric analysis of the spleens, demonstrated that the expression level of CCR3, the receptor for eo-2, was higher in atherosclerotic ApoE $\mathrm{KO}$ mice compared to their young littermates $(\mathrm{P}<0.01$; ANOVA) (Figure 1(b)).

\subsection{In Vitro Biological Effects of Anti-Eo-2 Antibodies}

We have generated monoclonal antibodies against human eo-2. Of our newly-developed monoclonal antibodies, D8 (at concentration of $50 \mathrm{ug} / \mathrm{ml}$ ) significantly attenuated adhesion of murine and rat splenocytes as well as human PBMCs to fibronectin by $35 \%$ - 55\% (Figure 2(a)) and also attenuated their migration towards VEGF in a dose dependent manner (Figure 2(b)). Migration and adhesion (VEGF and fibronectin, respectively) serve as potent proadhesive and promigratory properties yet regardless of eo-2 CCR3 interactions. The adhesion and migration data presented herein as well as in our previous study [19] lead us to assume that D8 effects maybe due to potential cross reactivity with other cellular receptors. The monoclonal antibody (at 5 and $50 \mathrm{ug} / \mathrm{ml}$ ) also inhibited adhesion to eo-2 of HEK cells stablytransfected with CCR3 (Figure 2(c)), as well as migration towards eo- 2 by $20 \%$ in comparison to CCR3transfected cells preincubated with total $\operatorname{IgG}$ (data not shown). Each one of the experiments described here was performed in six replicates and repeated twice. The results presented herein demonstrate that D8 interferes at least in part with the CCR3/eotaxin-2 binding interaction. The cross-reactivity of D8 with human and murine eotaxin-2 (5 ug eo-2 diluted in PBS), with $\mathrm{Kd}$ of $0.77 \mathrm{~g}$ and $4 \mathrm{~g}$, respectively, was verified.

\subsection{In Vivo Effects of Anti-Eo-2 Antibodies}

First we sought to assess whether eo-2 secretion is indeed present in advanced atherosclerotic plaques, in close proximity with intra-plaque inflammatory cells. To this end, we performed histological analysis of aortic plaques of young (6 weeks old) and old (4 months old) ApoE mice (according to the experience in our animal 


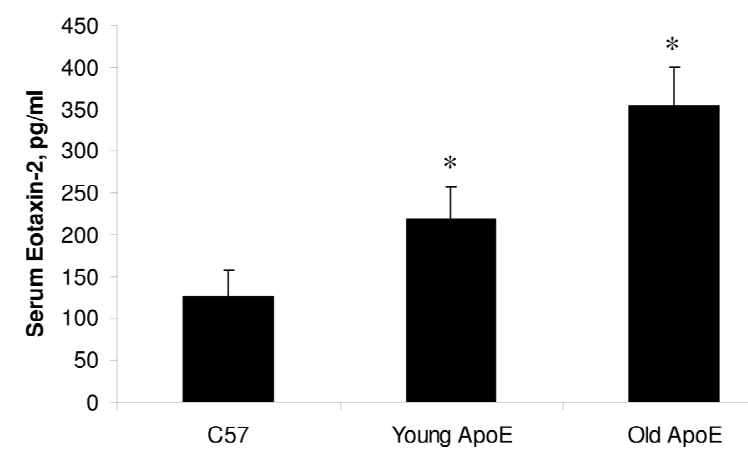

(a)
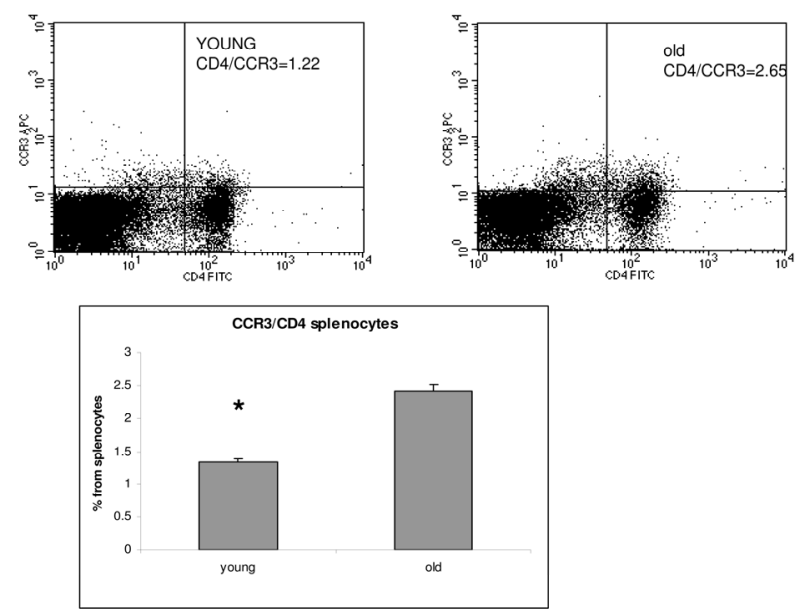

(b)

Figure 1. Overexpression of eo-2/CCR3 in old ApoE mice. Detection of eotaxin-2 in murine sera by ELISA (a) and CCR3 in murine splenocytes by FACS analysis (b). ${ }^{*} \mathrm{P}<0.05$.

house, this is the rate of progression of atherosclerosis in ApoE KO mice' though we do realize that in different animal houses, there may be differences in the rate of progression of experimental atheroma).

Indeed an IHC analysis of aortic frozen sections revealed expression of eo-2 in intermediate or advanced lesions (Figures 3(a) and (b), respectively), but not in early lesions isolated from 6 weeks old mice (Figure 3(c)). These untreated advanced lesions were significantly positive for macrophage staining (Figure 4(A)) and were also found positive for $\mathrm{T}$ cell staining (Figure 4(D)). Interestingly, the inflammatory cells were significantly reduced, in aortas of mice treated with D8 (twice weekly, for total course of 8 weeks) (Figures 4(C) and (F)) and only partly missing in IgG-treated animals (Figures 4(B) and (E)). Based on the encouraging data, we sought to study the effects of anti-eo-2 antibodies on plaque phenotype in young (6 weeks old) and old (4 months old) ApoE KO mice. In the first preliminary study, we have found that administration of commercial polyclonal anti-eo-2 antibodies twice weekly at doses of $5 \mathrm{ug}$ was efficient in reducing fatty-streaks in 4 young ( 6 weeks old) ApoE KO mice already after three weeks com-

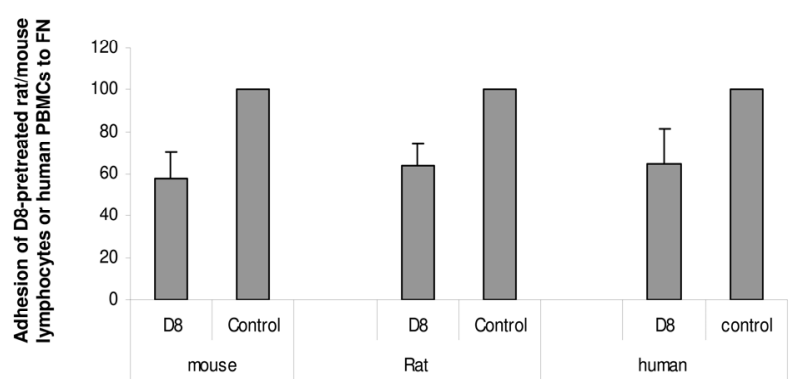

(a)

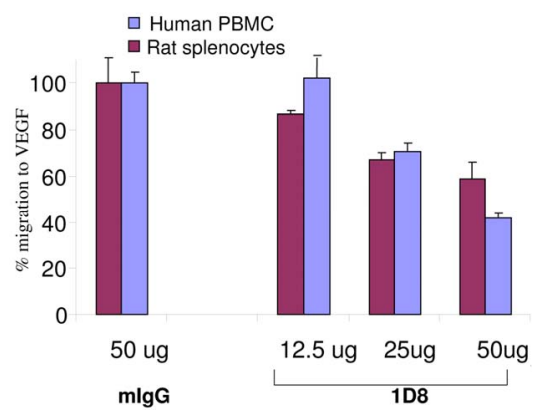

(b)

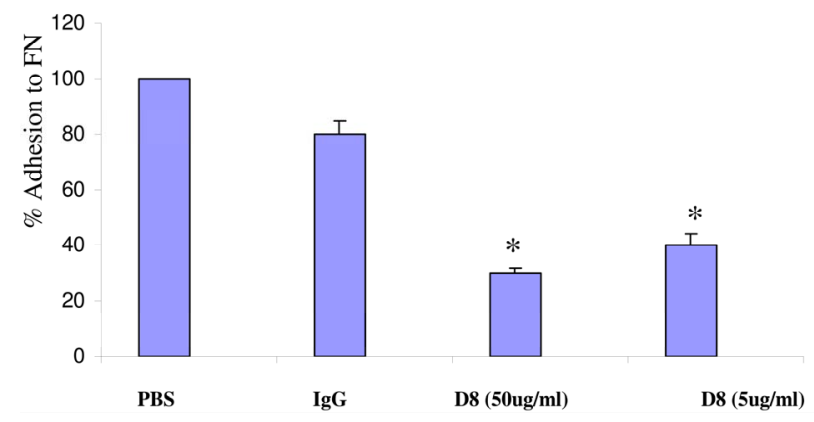

(c)

Figure 2. Functional properties a monoclonal antibody to eo-2 (D8). (a) and (b) Lymphocytes from murine, rat and human PBMC recovered via ficoll gradient were allowed to adhere to $10 \mathrm{~cm}$ tissue culture dish for an overnight incubation. The cells were then trypsinized and preincubated with D8 or murine IgG for 2 hours. (a) Inhibition of adhesion to Fibronectin (FN). Percent adhesion to FN of D8 (50 g)-treated compared to mIgGtreated lymphocytes; (b) Inhibition of migratory properties. Percent migration towards VEGF of D8-treated compared to mIgG-treated lymphocytes; (c) Interference with CCR3/eotaxin-2 interactions. Human-CCR3 tansfected HEK cells were plated on 96-well plates, pre-coated with eotaxin-2 $(5 \mathrm{ug} / \mathrm{ml}) /$ $1 \mathrm{D} 8(30 \mathrm{ug} / \mathrm{ml})$ complexes or with eo-2 only. After one hourincubation, non-adherent cells were washed away and the amount of adherent cells was analyzed $\left({ }^{*} \mathrm{P}<0.05\right)$.

pared to control (vehicle only) treated animals (not shown). We thus used this dosage in designing two additional studies. The first was intended to assess the effect of administration of the same anti-eo- 2 commercial polyclonal antibodies to young ApoE KO mice compared to control mouse IgG on formation of fatty streaks. In this study, after eight weeks of treatment, we found that anti-eo-2 antibodies, significantly reduced fatty streak 


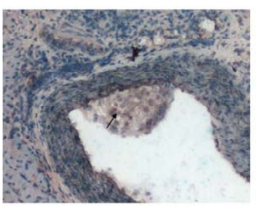

(a)

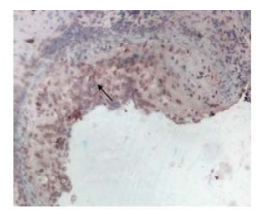

(b)

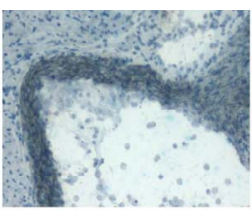

(c)
Figure 3. Immunohistochemical localization of eo-2 in murine atheroma. Fatty streaks and advanced lesions from 4 months old ApoE KO mice were stained with anti-eo-2 antibodies as described in methods. (a) shows a characteristic fatty streak of an intermediate plaque (AHA class II), (b) shows an atheromatous lesion (AHA class III-IV) and (c) shows a negative control of an early lesion stained with an irrelevant IgG.

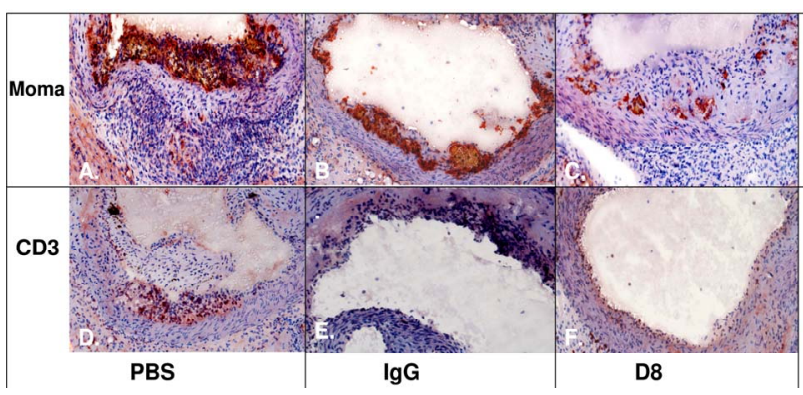

Figure 4. Macrophage and $\mathrm{T}$ cells infiltration is significantly reduced upon treatment with D8 compared to controls (PBS or $\mathrm{IgG}$ ). Panels (A)-(C) Macrophage staining with moma antibody; Panels (D)-(F) Staining for T lymphocytes using an antiCD3 antibody. Arrow-positive staining for eo-2.

formation in the young ApoE $\mathrm{KO}$ animals compared to mouse IgG by approximately 68 percent as demonstrated by oil-red $\mathrm{O}$ staining of the plaques $(\mathrm{P}=0.0038$, twotailed student's t-test) (Figures 5(a) and 6(a)-(c)). This effect was not associated with a change in total cholesterol and triglyceride levels that were similar in both groups (data not shown). Moreover, treatment with control murine IgG did not influence plaque progression in comparison with PBS injections.

In the second study, we have tested the effects of eo-2 blockade in 4 months old ApoE KO mice in which plaque architecture is more complex. Herein, after eight weeks of twice weekly injections of eo- 2 antibodies, plaque size, was decreased by $20 \%$ compared to the plaques of the control IgG and the difference was not statistically significant ( $\mathrm{P}=0.3$ ) (Figures 5(a) and 6(d)-(f)). However, when plaque phenotype in the old animals was measured by fibrous content as documented by Masson's trichrome, we found that antibodies to eo- 2 induced a more stable plaque phenotype evidenced by a larger fibrous area and a smaller lipid core (Figures 5(c) and 6(g)-(i)) $(\mathrm{P}=0.0167$ and 0.097 compared to vehicle control and $\mathrm{IgG}$, respectively; two-tailed student's t-test). By employing a different analysis tool, we calculated the percentage of samples in which fibrous cap was evident. The protective cap was evident in $24 \%, 41 \%$ and $56 \%$ of the vehicle-control, IgG and anti-eo-2 plaque sections

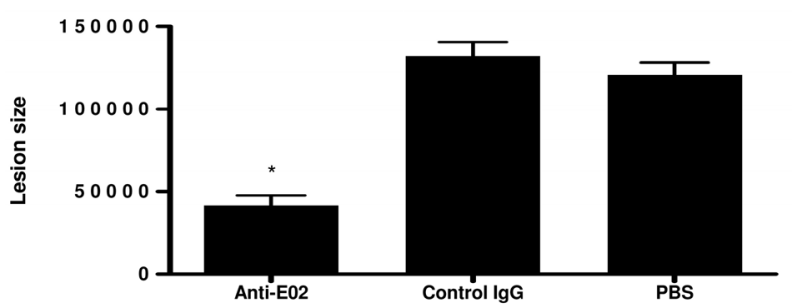

(a)

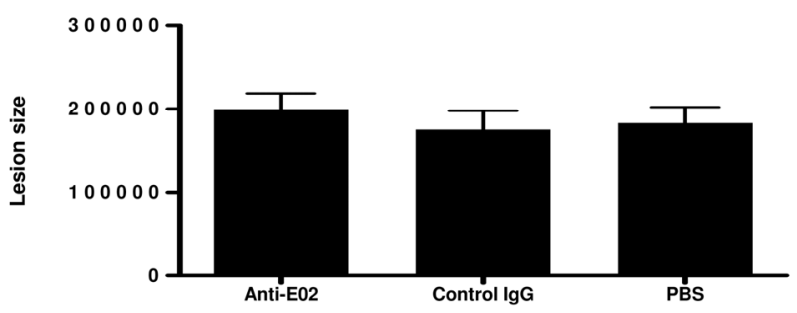

(b)

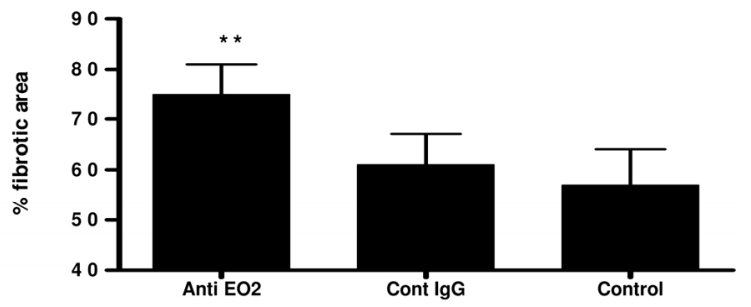

(c)

Figure 5. Treatment with blocking antibodies to eo-2 attenuates fatty streak formation and stabilizes advanced plaques (bar graph). Young ApoE KO mice treated with eo-2 antibodies, control IgG or PBS for eight weeks were sacrificed and their hearts were removed for assessment of plaque size using oil-red $\mathrm{O}$ staining. The bar graphs representing the staining intensity corresponding to plaque size are demonstrated in (a). Older ApoE KO mice were treated similarly after which atherosclerotic plaques size was analyzed by oil-red $\mathrm{O}(\mathrm{b})$ and the fibrous area evaluated by masson's trichome (c). ${ }^{*} \mathrm{P}<0.01 ;{ }^{* *} \mathrm{P}<0.05$.

( $\mathrm{P}<0.05$; two-tail student's t-test). Furthermore, the per centage of foam cell aggregates observed by Masson's trichrome staining was substantially lower in the anti eo-2 group $(81 \%, 71 \%$ and $56 \%$ in the PBS, IgG and anti-eo-2 groups, respectively; $\mathrm{P}<0.05)$. The data were confirmed in von Giesson's stain for elastic fibers (Figures 6(j)-(l)).

These findings were irrespective of the lipid levels that did not differ between groups. The IHC data point to the potential beneficial effects of anti eo- 2 antibodies in reducing the plaque size as well as increasing the plaque stability. A brief summary of the experimental treatment groups is given in Table $\mathbf{1}$.

\subsection{Effects of D8 Monoclonal Anti Eotaxin-2 Antibody on Atherosclerosis}

In view of the encouraging in vivo data demonstrated with the commercial polyclonal anti-eo- 2 antibody, we 


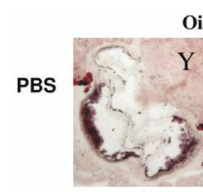

(a)

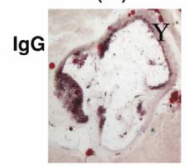

(b)

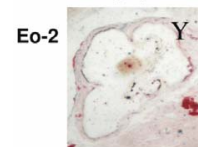

(c)

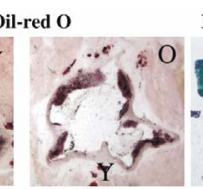

(d)

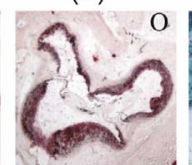

(e)

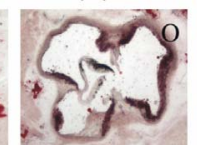

(f)

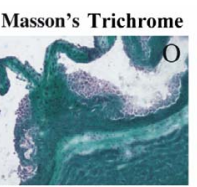

(g)

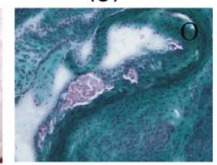

(h)

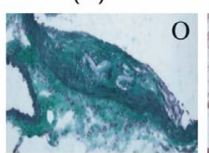

(i)

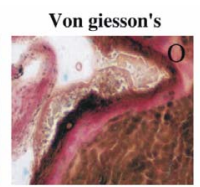

(j)

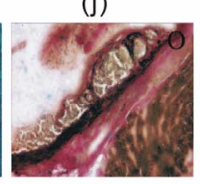

(k)
Figure 6. Representative sections from ApoE KO mice treated with blocking antibodies to eo-2. (a)-(c) show representative oil-red $\mathrm{O}$ stained fatty streak lesions from PBS, control IgG and eo-2 antibody treatment of young ApoE mice, respectively. Sections (d)-(f) show similar assessment of the more advanced lesions in older animals with the similar groups of mice. Sections (g)-(i) and (j)-(1) show Masson's trichrome and von Giesson's histochemistry (respectively) studies from either PBS, control IgG or eo-2antibody treatments, respectively in the older animals. Y-young; O-old.

sought to assess the potential therapeutic effects of our novel anti-eo-2 monoclonal antibodies. 6 - 8 weeks old ApoE KO mice were treated with D8 or with control IgG (30 ug, thrice weekly) for two months. Oil red O staining of the atherosclerotic plaques demonstrated a significant reduction in the atherosclerotic plaques in the D8 compared to the PBS or IgG-treated animals as demonstrated in Figure 7 ( $\mathrm{P}<0.05$, two-tail student's t-test).

\section{DISCUSSION}

Several research groups have recently suggested a role for chemokines and their receptors in the initiation and progression of atherosclerosis [20-25].

Murine and human atherosclerosis harbor abundant inflammatory cells and the role of the immune system in the process is well recognized $[11,14,15]$. It has already been reported that mast cells contribute importantly to allergic and innate immune responses by releasing various mediators [20]. Moreover, a direct participation of perivascular mast cells in atherogenesis and plaque destabilization has been documented in low-density lipoprotein receptor-deficient Ldlr (-/-) as well as in ApoE $\mathrm{KO}-/-[21]$ mice and also in human atherosclerotic plaques $[22,23]$. In addition, Halley et al have demonstrated that the CCR3 receptor localizes primarily to macrophage-rich regions in the human atheroma [22]. Data obtained using knockout mice showed a key role for the CC chemokine ligand 2 (CCL2, MCP1) and its receptor in the initiation of atherosclerosis [24] as well as for RANTES (CCL5) [25], pointing to the potential
Table 1. Summary of treatment groups.

\begin{tabular}{ccccc}
\hline $\begin{array}{c}\text { Duration } \\
\text { of } \\
\text { treatment }\end{array}$ & $\begin{array}{c}\text { Frequency of } \\
\text { treatment/week }\end{array}$ & $\begin{array}{c}\text { No. of } \\
\text { animals/group }\end{array}$ & Age & Treatment \\
\hline 8 weeks & 2 & 10 & $\begin{array}{r}4 \text { months } \\
6 \text { weeks \& }\end{array}$ & Commercial \\
8 weeks & 2 & 6 & $\begin{array}{r}4 \text { months } \\
\text { anti-eo-2 }\end{array}$ \\
8 weeks & 2 & 10 & $\begin{array}{c}6 \text { weeks \& } \\
4 \text { months }\end{array}$ & IgG \\
& 2 & 10 & $\begin{array}{c}6 \text { weeks \& } \\
4 \text { months }\end{array}$ & PBS \\
\hline weeks & 2 & & & \\
\hline
\end{tabular}

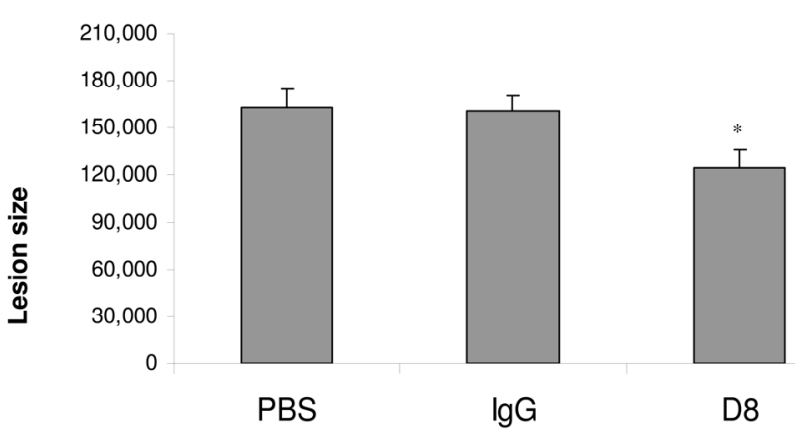

Figure 7. Treatment with D8 attenuates plaque size in young ApoE mice (bar graph). ApoE KO mice were treated with either monoclonal antibodies to eo-2, total mouse $\operatorname{IgG}$ or PBS as described in methods. Following 8 weeks of treatment, mice were sacrificed and their hearts removed for assessment of lesions size after oil-red $\mathrm{O}$ staining. Quantitative assessment of lesion size was performed as described. ${ }^{*} \mathrm{P}<0.05$.

involvement of these pathways in the initiation and progression of atherosclerosis. The potential involvement of the eo-2/CCR3 signaling pathway was greatly supported in our recent study in which we have demonstrated a significant effect of our newly-developed anti-eo-2 monoclonal antibody in a rheumatoid arthritis rat model [19].

Despite the apparent specificity of eo- 2 as well as eotaxin-1 to eosinophils, there is abundant data on the pleiotropic effects of these chemokines. Both eo- 1 and eo-2 are expressed in various types of endothelial cells [5,7-9] and induce migratory responses in endothelial $[8,9]$ and smooth muscle cells [13]. We have found that serum eo-2 levels as well as CCR3 expression level on splenocytes were more abundant in the old atherosclerotic ApoE KO mice compared with the younger litters that exhibit only initial fatty streaks. A neutralizing polyclonal antibody as well as one of our newly-produced monoclonal antibodies to eo-2 conferred anti-adhesive and anti-migratory properties as well as in vivo reduction of the atherosclerotic lesion progression. In the younger mice, the antibodies mainly reduced fatty streak accumulation, whereas in the older animals, in which the plaques were more advanced, a more stable phenotype of the atheroma was evident upon treatment.

There is an abundance of macrophages as well as $\mathrm{T}$ cells in advanced atherosclerotic lesions (those cells are 
the ones that highly express CCR3). These immune cells, however, were significantly reduced in lesions of the antibody-treated mice. The data highly suggest an important role for the immune system in general and specifically for the CCR3-eo-2 receptor-ligand interactions in the progression of atherosclerosis, in a similar manner as the data obtained in the rheumatoid arthritis model.

The data presented herein suggest that blocking inflammatory cell adhesion to the arterial endothelium may be principally responsible for those beneficial effects observed with the neutralizing blocking polyclonal and monoclonal eo-2 antibodies.

In conclusion, we have shown here for the first time that the chemokine eo- 2 plays a role in experimental atherosclerosis. These findings were demonstrated by showing that the atherosclerotic lesions of ApoE KO mice contain abundant eo-2, harbor higher levels of its receptor CCR3 and that blockade of this pathway results in attenuated in vitro migration of inflammatory cells with reduced experimental early atherogenesis and establishment of a more stable plaque phenotype.

If further confirmed, these findings may illuminate an additional interaction that governs atherosclerotic plaque growth and destabilization.

\section{REFERENCES}

[1] Jose, P.J., Griffiths-Johnson, D.A., Collins, P.D., Walsh, D.T., Moqbel, R., Totty, N.F., Truong, O., Hsuan, J.J. and Williams, T.J. (1994) Eotaxin: A potent eosinophil chemoattractant cytokine detected in a guinea pig model of allergic airways inflammation. The Journal of Experimental Medicine, 179, 881-887. doi:10.1084/jem.179.3.881

[2] Kitaura, M., Nakajima, T., Imai, T., Harada, S., Combadiere, C., Tiffany, H.L., Murphy, P.M. and Yoshie, O. (1996) Molecular cloning of human eotaxin, an eosinophil-selective CC chemokine, and identification of a specific eosinophil eotaxin receptor, $\mathrm{CC}$ chemokine receptor 3. The Journal of Biological Chemistry, 271, 7725-7730. doi:10.1074/jbc.271.13.7725

[3] Ponath, P.D., Qin, S., Ringler, D.J., Clark-Lewis, I., Wang, J., Kassam, N., Smith, H., Shi, X., Gonzalo, J.A., Newman, W., Gutierrez-Ramos, J.C. and Mackay, C.R. (1996) Cloning of the human eosinophil chemoattractant, eotaxin. Expression, receptor binding, and functional properties suggest a mechanism for the selective recruitment of eosinophils. Journal of Clinical Investigation, 97, 604612. doi:10.1172/JCI118456

[4] Pease, J.E. (2006) Asthma, allergy and chemokines. Current Drug Targets, 7, 3-12. doi:10.2174/138945006775270204

[5] Berger, O., Gan, X., Gujuluva, C., Burns, A.R., Sulur, G., Stins, M., Way, D., Witte, M., Weinand, M., Said, J., Kim, K.S., Taub, D., Graves, M.C. and Fiala, M. (1999) $\mathrm{CXC}$ and $\mathrm{CC}$ chemokine receptors on coronary and brain endothelia. Molecular Medicine, 5, 795-805.
[6] Salcedo, R., Resaum J.H., Halversonm D., Hudsonm E.A., Dambach, M., Powell, D., Wasserman, K. and Oppenheim, J.J. (2000) Differential expression and responsiveness of chemokine receptors (CXCR1-3) by human microvascular endothelial cells and umbilical vein endothelial cells. The FASEB Journal, 14, 2055-2064. doi:10.1096/fj.99-0963com

[7] Romagnani, P., Annunziato, F., Lasagni, L., Lazzeri, E., Beltrame, C., Francalanci, M., Uguccioni, M., Galli, G., Cosmi, L., Maurenzig, L., Baggiolini, M., Maggi, E., Romagnani, S. and Serio, M. (2001) Cell cycle-dependent expression of CXC chemokine receptor 3 by endothelial cells mediates angiostatic activity. Journal of Clinical Investigation, 107, 53-63. doi:10.1172/JCI9775

[8] Salcedo, R., Young, H.A., Ponce, M.L., Ward, J.M., Kleinman, H.K., Murphy, W.J. and Oppenheim, J.J. (2001) Eotaxin (CCL11) induces in vivo angiogenic responses by human CCR3+ endothelial cells. The Journal of Immunology, 166, 7571-7578.

[9] Cheng, S.S., Lukacs, N.W. and Kunkel, S.L. (2002) Eotaxin/CCL11 suppresses IL-8/CXCL8 secretion from human dermal microvascular endothelial cells. The Journal of Immunology, 168, 2887-2894.

[10] Agrawal, L., Maxwell, C.R., Peters, P.J., Clapham, P.R., Liu, S.M., Mackay, C.R. and Strayer, D.S. (2009) Complexity in human immunodeficiency virus type 1 (HIV-1) co-receptor usage: Roles of CCR3 and CCR5 in HIV-1 infection of monocyte-derived macrophages and brain microglia. Journal of General Virology, 90, 710-722. doi:10.1099/vir.0.006205-0

[11] Hansson, G.K. (2005) Inflammation, atherosclerosis, and coronary artery disease. The New England Journal of Medicine, 352, 1685-1695. doi:10.1056/NEJMra043430

[12] Sun, J., Sukhova, G.K., Wolters, P.J., Yang, M., Kitamoto, S., Libby, P., MacFarlane, L.A., Mallen-St Clair, J. and Shi, G.P. (2007) Mast cells promote atherosclerosis by releasing proinflammatory cytokines. Nature Medicine, 13, 719-724. doi:10.1038/nm1601

[13] Kodali, R., Hajjou, M., Berman, A.B., Bansal, M.B., Zhang, S., Pan, J.J. and Schecter, A.D. (2006) Chemokines induce matrix metalloproteinase-2 through activation of epidermal growth factor receptor in arterial smooth muscle cells. Cardiovascular Research, 69, 706-715. doi:10.1016/j.cardiores.2005.09.012

[14] Sheikine, Y., Olsen, B., Gharizadeh, B., Jatta, K., Tornvall, P. and Ghaderi, M. (2006) Influence of eotaxin $67 \mathrm{G}>\mathrm{A}$ polymorphism on plasma eotaxin concentrations in myocardial infarction survivors and healthy controls. Atherosclerosis, 189, 458-463. doi:10.1016/j.atherosclerosis.2006.01.003

[15] Economou, E., Tousoulis, D., Katinioti, A., Stefanadis, C., Trikas, A., Pitsavos, C., Tentolouris, C., Toutouza, M.G. and Toutouzas, P. (2001) Chemokines in patients with ischaemic heart disease and the effect of coronary angioplasty. International Journal of Cardiology, 80, 55-60. doi:10.1016/S0167-5273(01)00454-5

[16] Emanuele, E., Falcone, C., D’Angelo, A., Minoretti, P., Buzzi, M.P., Bertona, M. and Geroldi, D. (2006) Association of plasma eotaxin levels with the presence and 
extent of angiographic coronary artery disease. Atherosclerosis, 186, 140-145. doi:10.1016/j.atherosclerosis.2005.07.002

[17] Ardigo, D., Assimes, T.L., Fortmann, S.P., Go, A.S., H1atky, M., Hytopoulos, E., Iribarren, C., Tsao, P.S., Tabibiazar, R., Quertermous, T. and ADVANCE Investigators (2007) Circulating chemokines accurately identify individuals with clinically significant atherosclerotic heart disease. Physiological Genomics, 31, 402-409.

[18] Sheikine, Y.A. and Hansson, G.K. (2006) Chemokines as potential therapeutic targets in atherosclerosis. Current Drug Targets, 7, 13-27. doi: $10.2174 / 138945006775270240$

[19] Ablin, J.N., Entin-Meer, M., Aloush, V., Oren, S., Elkayam, O., George, J. and Barshack, I. (2010) Protective effect of eotaxin-2 inhibition in adjuvant-induced arthritis. Clinical \& Experimental Immunology, 161, 376-283. doi:10.1111/j.1365-2249.2010.04172.x

[20] Weber, C., Zernecke, A. and Libby, P. (2008) The multifaceted contributions of leukocyte subsets to atherosclerosis: Lessons from mouse models. Nature Reviews Immunology, 8, 802-815. doi:10.1038/nri2415

[21] Bot, I., de Jager, S.C., Zernecke, A., Lindstedt, K.A., van Berkel, T.J., Weber, C. and Biessen, E.A. (2007) Perivas- cular mast cells promote atherogenesis and induce plaque destabilization in apolipoprotein E-deficient mice. Circulation, 115, 2516-2525. doi:10.1161/01.ATV.14.9.1480

[22] Haley, K.J., Lilly, C.M., Yang, J.H., Feng, Y., Kennedy, S.P., Turi, T.G., Thompson, J.F., Sukhova, G.H., Libby, P. and Lee, R.T. (2000) Overexpression of eotaxin and the CCR3 receptor in human atherosclerosis: Using genomic technology to identify a potential novel pathway of vascular inflammation. Circulation, 102, 2185-2189. doi:10.1161/01.CIR.102.18.2185

[23] Kovanen, P.T. (2007) Mast cells: Multipotent local effector cells in atherothrombosis. Immunological Reviews, 217, 105-122. doi:10.1111/j.1600-065X.2007.00515.x

[24] Boring, L., Gosling, J., Cleary, M. and Charo, I.F. (1998) Decreased lesion formation in CCR2-/- mice reveals a role for chemokines in the initiation of atherosclerosis. Nature, 394, 894-897. doi:10.1038/29788

[25] Hillyer, P., Mordelet, E., Flynn, G. and Male, D. (2003) Chemokines, chemokine receptors and adhesion molecules on different human endothelia: Discriminating the tissue-specific functions that affect leucocyte migration. Clinical \& Experimental Immunology, 134, 431-441. doi:10.1111/j.1365-2249.2003.02323.x 